\title{
Mathematical model for acquiring immunity to malaria: a PDE approach
}

\author{
S. Y. Tchoumi ${ }^{1}$, Y. T. Kouakep ${ }^{2}$, D. J. M. Fotsa ${ }^{1}$, F. G. T. Kamba ${ }^{3}$, \\ J. C. Kamgang ${ }^{1}$, D. D. E. Houpa ${ }^{3}$
}

${ }^{1}$ Department of Mathematics and Computer Sciences

ENSAI - University of N'Gaoundéré, P.O.Box 455 N'Gaoundéré, Cameroon

2 Department of Basic and Technical Engineering Sciences

EGCIM - University of N'Gaoundéré, P.O.Box 454 N'Gaoundéré, Cameroon

kouakep@aims-senegal.org

${ }^{3}$ Department of Mathematics and Computer Sciences, Faculty of Sciences

University of N'Gaoundéré, P.O.Box 454 N'Gaoundéré, Cameroon

Received: 3 December 2020, accepted: 22 July 2021, published: 12 September 2021

\begin{abstract}
We develop a new model of integro-differential equations coupled with a partial differential equation that focuses on the study of the naturally acquiring immunity to malaria induced by exposure to infection. We analyze a continuous acquisition of immunity after infected individuals are treated. It exhibits complex and realistic mechanisms precised mathematically in both disease free or endemic context and in several numerical simulations showing the interplay between infection through the bite of mosquitoes. The model confirms the (partial) premunition of the human population in the regions where malaria is endemic. As common in literature, we indicate an equivalence of the basic reproduction rate as the spectral radius of a "next generation" operator.
\end{abstract}

Keywords-Malaria, Premunition, modeling, Endemic

MSC2010: 35K20, 92B05

\section{INTRODUCTION}

One of the major health challenges in Africa is the management of malaria endemicity [15], [9], [13], [21], [11], [3], [15], [18]. As underlined by Langhorne [11] "preventative and treatment strategies are continuously hampered by the issues of the ever-emerging parasite resistance to newly introduced drugs, considerable costs and logistical problems". Understanding the mechanisms of naturally acquiring immunity to malaria [11] even with modeling, is important to analyze its hidden mecha-

Copyright: (C) 2021 Tchoumi et al. This article is distributed under the terms of the Creative Commons Attribution License (CC BY 4.0), which permits unrestricted use, distribution, and reproduction in any medium, provided the original author and source are credited.

Citation: S Y Tchoumi, Y T Kouakep, D J M Fotsa, F G T Kamba, J C Kamgang, D D E Houpa, Mathematical model for acquiring immunity to malaria: a PDE approach, Biomath 10 (2021), 2107227, http://dx.doi.org/10.11145/j.biomath.2021.07.227 
nisms, and justifies our study [19], [9], [13], [21], [11], [3], [15], [18]. The "premunition" could be defined as the natural acquired immunity capacity to live with a relatively low concentration of malaria parasite, and resist to falling sick. The immunity is not a sterilizing type in that the infection persists longer than the symptoms and individuals can exhibit relapses or recrudescences or become reinfected. Moreover, chronic infection persists, although the maximum parasite load reached is low. Even if it adds a little in terms of reduction of parasite load as compared to innate resistance, this additional immunity is substantial in terms of morbidity as it keeps the parasite load low, below the threshold of pathogens. Super infection can occur, but it remains at a low grade" [19]. Obi et al. [19] precised that "premunition is independent of transmission levels provided it occurs at least once a year". It is rapidly lost: exactly one year without re-challenge is enough to lose this protective state. It is strain independent and clearly immunoglobulin G (IgG) dependent. The delay of acquisition is remarkably long, compared to the rate of transmission". Moreover, [19] states that epidemiological studies in Africa and Papua New Guinea have helped to define three clinical periods: a short period of 0 - 5 years where mortality can occur; a long period of 0 to $15-20$ years where morbidity is "frequent" (though decreaing in frequency with age); thereafter a longer period of premunition where the diseases in any form is absent. This paper addresses the impact of a continuous level of premunition on a scale of 0 (non immune) to 1 (high degree of immune responsiveness to infection in terms of premunition) in recovered individuals. It will be interesting to see which mechanisms support the evolution of the number of (partially) immune humans to the natural acquisition of premunition. It is common to see that an individual from an endemic region (central Africa, South America..) who goes out to a malaria epidemic region (west Europe for e.g), has a great chance to fall sick if he turns back to another endemic (malaria) region [22]. [14] claims that "after a couple of more infections, anti-disease immunity develops and causes suppression of clinical symptoms even in the presence of heavy parasitemia and also reduces the risk of severe disease. Frequent and multiple infections slowly lead to the development of anti-parasite immunity that results in very low or undetectable parasitemia. (...). Premunition suggests an immunity mediated directly by the presence of the parasites themselves and not as much as the result of the previous infections" [13], [9]. Malaria affects more than $40 \%$ of the world population in over 90 countries [14]. We adopt the partial differential approach to model the premunition acquisition. More specifically, we use an integro-differential modeling coupled with a partial differential equation because they track very well the continuity of the temporary immunity level. One could rather consider several discrete states of the immunity level and obtain a huge number of differential equations that are more complex to use. After some mathematical analysis of the model, we made several numerical simulations. Our mathematical analysis and simulations support the fact that the premunition is a continuous process because the relative resistance to a low level of parasite seems to increase with time in an endemic situation. But it is clear that the level of premunition is somehow a probability with a possibility to suffer from the severe malaria disease if other negative factors are dramatically considered (physiological lacks, physical or psychological injuries ...) even if the level of premunition is high. Moreover, the non frequently mosquitoes-human biting contact like in the use (properly or not) of the bed 
nets could lead to a partial immunity with a possibility to suffer from the severe malaria disease. As a biological and modeling assumption, we consider that usage of bed net should be rigorous to avoid instability in the premunition acquisition process. Following [14], [13], [9], more recovered people in endemic area seems to have a high degree of immune responsiveness after being continuously exposed to mosquitoes biting. Then, in endemic areas, the adult population (of more than 5 years) could live with the parasite in an asymptomatic carriers status. It explains why public health strategies in high endemicity region of malaria, concerns children from 0 to 5 years old and pregnant women. Our main result supports the fact that more recovered people in endemic area seem to have a high degree of immune responsiveness after being continuously exposed to mosquitoes biting. Finally, it's likely to see that in malaria endemic areas, the population (in adulthood likely more that five years old) could live mainly as "recovered" even within the presence of heavy parasitemia as biologically pointed out by [14], [13], [9]. Our work is subdivided as follow: The second section describes our model and some extensions, the third section presents the main results. The fourth section shows the simulations and analyses them, mainly in the cases where the transmission functions $a, m, c, \tilde{c}(., \theta)$ are periodic (even constant). The fifth section discuss the important results and in the last section, we conclude our work.

\section{Model Description AND EXTENSion}

Similarly as in [8], [18], the model subdivides the total human population at time $t$, denoted by $N_{h}(t)$, into the following sub-populations of susceptible $S_{h}(t)$, symptomatic infectious with sickness $I_{h}(t)$ and recovered individuals $R_{h}(t, \theta)$ with temporal immunity level $\theta \in[0,1]$ at a time $t \geq$ 0 . Biologically, it is logical to assume that the level of acquired immunity cannot be 0 in endemic areas like Africa. So that $N_{h}(t)=S_{h}(t)+I_{h}(t)+\int_{0}^{1} R_{h}(t, \theta) d \theta$. The total vector (mosquito) population at time $t$, denoted by $N_{v}(t)$, is sub-divided into susceptible $S_{v}(t)$ and infectious mosquitoes $I_{v}(t)$. Thus, $N_{v}(t)=S_{v}(t)+I_{v}(t)$. Susceptible individuals are recruited at a constant rate $\Gamma_{h}(t)$. We define the force of infection from mosquitoes to humans by $\beta(t)$ as the product of the transmission rate per contact with infectious mosquitoes $m(t)$ and the successful biting rate after a contact $a(t)$ (seen in [8] as the product of the mosquito contact rate $\alpha$ with the mosquito biting rates $\theta_{m} h(t)$ ) and the probability that the mosquito is infectious $I_{v} / N_{h}$ [18]. The natural death rate of human is $\mu_{h}$. Recovered individual loses immunity at a rate $\gamma(t, \theta)$. Susceptible mosquitoes are generated at a per capita rate $\Gamma_{v}(t)$ at time $t$ and acquire malaria through contacts with infectious humans with the force of infection $\varphi(t)$. Mosquitoes are assumed to suffer death due to natural causes at a rate $\mu_{v}(t)$ at time $t . g(t, \theta) R_{h}(t, \theta)$ represents the total number of recovered leaving the level $\theta$ to the greater level $\theta^{\prime \prime} \in$ ]$\theta, 1]$ and $g(t, \theta)<\gamma(t, \theta)$.

We emphasize on the term $R_{h}(t, \theta)$ which is the number of recovered individuals with an acquired temporal immunity level $\theta \in$ $[0,1]$ at a time $t \geq 0$. It's dynamics is described by

$$
\frac{\partial R_{h}(t, \theta)}{\partial t}+\frac{\partial j(\theta) R_{h}(t, \theta)}{\partial \theta}
$$

$$
=\beta(t) \int_{0}^{\theta} R_{h}\left(t, \theta^{\prime}\right) d \theta^{\prime}-\gamma(t, \theta) R_{h}(t, \theta)
$$

that includes through $\beta(t)$, the impact of the "new bites of mosquitoes" on all the $R_{h}(t, s)$ 'recovered individuals who moves to a next stage of new individuals with a greater level of immunity $\theta>s$. 
S Y Tchoumi et al., Mathematical model for acquiring immunity to malaria: a PDE ...

\section{A. Model equation}

The formulation and construction of the model's equations (for $t, \theta>0$ ) are given by:

$$
\left\{\begin{aligned}
\frac{d S_{h}(t)}{d t}= & \Gamma_{h}(t)+\int_{0}^{1}\left(\gamma\left(t, \theta^{\prime}\right)-g(t, \theta)\right) R_{h}\left(t, \theta^{\prime}\right) d \theta^{\prime} \\
& -\beta(t) S_{h}(t)-\mu_{h}(t) S_{h}(t) \\
\frac{d I_{h}(t)}{d t}= & \left.\beta(t) S_{h}(t)-\delta(t)\right) I_{h}(t)-\tilde{\mu}_{h}(t) I_{h}(t) \\
\frac{\partial R_{h}(t, \theta)}{\partial t}+ & +\frac{\partial j(\theta) R_{h}(t, \theta)}{\partial \theta}= \\
& \beta(t) \int_{0}^{\theta} R_{h}\left(t, \theta^{\prime}\right) d \theta^{\prime}-\gamma(t, \theta) R_{h}(t, \theta) \\
\frac{d S_{v}(t)}{d t}= & \Gamma_{v}(t)-\left(\varphi(t)+\mu_{v}(t)\right) S_{v}(t) \\
\frac{d I_{v}(t)}{d t}= & \varphi(t) S_{v}(t)-\mu_{v}(t) I_{v}
\end{aligned}\right.
$$

where $\beta(t)=\frac{a(t) m(t) I_{v}(t)}{N_{h}(t)}, j(\theta)=\alpha \theta$ and

$\varphi(t)=\frac{a(t)\left(c(t) I_{h}(t)+\int_{0}^{1} \tilde{c}\left(t, \theta^{\prime}\right) R_{h}\left(t, \theta^{\prime}\right) d \theta^{\prime}\right)}{N_{h}(t)}$

with $\tilde{\mu}=\mu_{h}+d$ and (1) initial conditions $\left(P_{1}\right)$ or $\left(P_{2}\right)$ exclusively, that is

$$
\left(P_{1}\right)\left\{\begin{array}{l}
S_{h}(0)=S_{h}^{0} \\
I_{h}(0)=I_{h}^{0} \\
R_{h}(t, 0)=\delta(t) I_{h}(t) \\
R_{h}(0, \theta)=R_{h}^{0}(\theta), \forall \theta \in[0,1] \\
I_{v}(0)=I_{v}^{0} \\
S_{v}(0)=S_{v}^{0}
\end{array}\right.
$$

or

$$
\left(P_{2}\right)\left\{\begin{array}{l}
S_{h}(0)=S_{h}^{0} \\
I_{h}(0)=I_{h}^{0} \\
\frac{\partial R_{h}(t, 0)}{\partial t}=\delta(t) I_{h}(t) \\
R_{h}(0, \theta)=R_{h}^{0}(\theta), \forall \theta \in[0,1] \\
I_{v}(0)=I_{v}^{0} \\
S_{v}(0)=S_{v}^{0} .
\end{array}\right.
$$

Remark 2.1: The function $j(\theta)$ is a factor characterizing the rate of entering the $R_{h}(t, \theta)$ compartment if other influxes are neglected. A generalization of the function $j$ could include the state of a withinhost model of blood cells with the malaria pathogen [16].

The function $g(t, \theta)$ could be $\beta(t) z(\theta)$, but one could also consider that $g(t, \theta)=(1-$ k) $\gamma(t, \theta)$ with $k \in[0,1]$ as in the section IV of simulations. The first and third equation re-stated become:

$$
\left\{\begin{array}{c}
\frac{d S_{h}(t)}{d t}=\Gamma_{h}(t)+\int_{0}^{1} k \gamma\left(t, \theta^{\prime}\right) R_{h}\left(t, \theta^{\prime}\right) d \theta^{\prime} \\
-\beta(t) S_{h}(t)-\mu_{h}(t) S_{h}(t) \\
\frac{\partial R_{h}(t, \theta)}{\partial t}+\frac{\partial j(\theta) R_{h}(t, \theta)}{\partial \theta}= \\
\beta(t) \int_{0}^{\theta} R_{h}\left(t, \theta^{\prime}\right) d \theta^{\prime}-\gamma(t, \theta) R_{h}(t, \theta),
\end{array}\right.
$$

where $k$ represents the fraction of recovered individuals $R(t, \theta)$ who move to other levels $\left.\left.\theta^{\prime} \in\right] \theta, 1\right]$.

We will explore some of this particular cases:

Case 1. Values of $\gamma, g$ and $\tilde{c}$ motivated by the biological references therein:

- the level of infection $\theta$ satisfies $\frac{d \theta}{d t}=\alpha \theta$ and $\int_{0}^{1} \rho(\theta)=1$, with $\rho$ a probability density [26], [24]

$$
\rho_{k, l}(\theta)=l \frac{(-l \cdot \ln (\theta))^{k-1} \theta^{l-1}}{\Gamma(k)} e^{-l \theta} .
$$

- Consider

$$
g(t, \theta)=(1-k) \cdot \gamma(t, \theta)
$$

where

$$
\gamma(t, \theta)=\gamma(t)=\frac{h e^{-h t}}{1-e^{-h t}},
$$

$h$ is the annual rate of infections of individuals [12], $0<k \leq 0.4466$ and $0.4466=$ $76.6 \times 58.3$ is the probability to be likely protected from the common perfect possession and use of the bed nets in Cameroon [2], [5] over three years of use of the Long-lasting insecticide-treated bed nets (LLINs). We have the average 
S Y Tchoumi et al., Mathematical model for acquiring immunity to malaria: a PDE ...

TABLE I: Table of parameters

\begin{tabular}{|c|c|c|c|}
\hline Parameters & Interpretation & Average value & Reference \\
\hline$e$ & immigration rate of humans & $\frac{12}{365.25}$ & [7] \\
\hline$f$ & relative birth rate of humans & $\frac{40}{36520}$ & [7] \\
\hline$j$ & relative rate of acquiring premunition & & {$[16$} \\
\hline$q$ & immigration rate of mosquitoes & $\frac{10000}{21}$ & [5] \\
\hline$x$ & birth rate of new adult female mosquitoes & $\frac{21}{130}$ & [7], [4] \\
\hline$\mu_{h}$ & humans per capita death rate & $\frac{1000}{59 \times 365.25}$ & modified [7], [8] \\
\hline$\mu_{v}$ & mosquitoes per capita death rate & $\frac{1}{21}$ & [7], [18] \\
\hline$\Gamma_{v}(t)$ & recruitment of mosquitoes & $q+x . N_{v}(t)$ & [7] \\
\hline$\Gamma_{h}(t)$ & recruitment of humans & $e+f . N_{h}(t)$ & [7] \\
\hline$a(t)$ & man biting rate & $0.5 \times 19$ & [7], [1] \\
\hline$m(t)$ & prob. of dis. transm. from inf. mosq. to human & 0.022 & [7] \\
\hline$c(t)$ & prob. of dis. transm. from inf. humans to mosq. & 0.48 & [7] \\
\hline$\tilde{c}(t)$ & prob. of dis. transm. from recovered humans to mosq. & 0.048 & [7] \\
\hline$d(t))$ & human disease induce mort.rate & $9 \times 10^{-5}$ & [1] \\
\hline$\gamma(t, \theta)$ & average per cap. rate of lost of immu. & {$[0.027-0.0146]$} & [1] \\
\hline $\begin{array}{l}h \\
\delta\end{array}$ & $\begin{array}{l}\text { rate of infectious per year for } S_{h} \text { to become } I_{h} \\
\text { recovery rate of humans }\end{array}$ & $\begin{array}{c}h=\frac{\ln \left(\frac{1}{1-e^{180 .\langle\gamma\rangle}}\right)}{180} \\
{[0.035-0.03704]}\end{array}$ & {$[12$} \\
\hline
\end{tabular}

value $\langle\gamma\rangle=\frac{1}{180} \int_{0}^{180} \gamma(t) d t$ (180 days is estimated by Raoult [22]) of $\gamma$ that leads to

$$
h=\frac{\ln \left(\frac{1}{1-e^{180 .\langle\gamma\rangle}}\right)}{180} .
$$

See values of $\langle\gamma\rangle$ in Table I. It is also possible to consider the average value as $\gamma$ (seen as constant);

- $\tilde{c}(t, \theta)$ could be either a constant or $c(t) \rho(\theta)$.

Case 2. $\Gamma_{h}(t)=e+f(t) S_{h}(t)$ and $\Gamma_{v}(t)=q+$ $x(t) S_{v}(t)$. Here, these forms combines the proportional and constant influxes (birth, migration, ...) in the humans and mosquitoes compartments.

Case 3. $\Gamma_{h}(t)=\Gamma_{h}>0$ and $\Gamma_{v}(t)=\Gamma_{v}>0$ seen as constants.

Case 4. In the references below, we collect these values of the parameters:

This kind of models which track the global dynamics according to the temporal level of immunity (inducing premunition) has not been developed before. Langhorne, Pinkevych and Mandal's reviews [11], [12], [21] described the lost of immunity in a local aspect (some discrete values of $\theta$ ) by focusing only on the dynamics of infected individuals. We go beyond that by studying continuously the impact of the reverse effect of acquiring immunity not discretely (for a fixed $\theta \in[0,1]$ ) but globally with all the interactions between the different individuals (susceptible or infected at all the stages of premunition) in order to see the overall dynamics.

\section{B. Extension of model (1) to different} malaria strains with same initial conditions

We shall consider a single strain of malaria (such as Plasmodium falciparum) in this study since it is the major cause of mortality and morbidity in the tropical and sub-tropical areas of the globe [23]. Several strains exists (falciparum, gambiae, coustani, balabacensis, funestus, nili, ...) [7, p.1290, Table A.3] but we focus on the afore mentioned. If we name " $i$ " the strain in a decreasing level of infectiousness depending on the global prevalence (with "1" for P. Falciparum, "2" for the next..., (i), ... until the strain " $n$ " with the lowest infectiousness and prevalence. $i$ could also be the patch index where the dominant strain is also called $i$ ), 
S Y Tchoumi et al., Mathematical model for acquiring immunity to malaria: a PDE ...

one could get the following model:

$$
\begin{aligned}
& \left\{\begin{aligned}
\frac{d S_{h}(t)}{d t}= & \Gamma_{h}(t) !+\Sigma_{1}^{n} \int_{0}^{1} k \gamma_{i}\left(t, \theta^{\prime}\right) R_{h}^{(i)}\left(t, \theta^{\prime}\right) d \theta^{\prime} \\
& -\Sigma_{1}^{n} \beta_{i}(t) S_{h}(t)-\mu_{h}(t) S_{h}(t)
\end{aligned}\right. \\
& \left.\frac{d I_{h}(t)}{d t}=\Sigma_{1}^{n} \beta_{i}(t) S_{h}(t)-\delta(t)\right) I_{h}(t)-\tilde{\mu}_{h}(t) I_{h}(t) \\
& \left\{\begin{array}{l}
\frac{\partial R_{h}^{(i)}(t, \theta)}{\partial t}+\frac{\partial \alpha \theta R_{h}^{(i)}(t, \theta)}{\partial \theta}= \\
\beta_{i}(t) \int_{0}^{\theta} R_{h}^{(i)}\left(t, \theta^{\prime}\right) d \theta^{\prime}-\left[\gamma_{i}(t, \theta)+\beta_{i}(t) g(\theta)\right] R_{h}^{(i)}(t, \theta)
\end{array}\right. \\
& \frac{d S_{v}(t)}{d t}=\Gamma_{v}(t)-\left(\Sigma_{1}^{n} \varphi_{i}(t)+\mu_{v}(t)\right) S_{v}(t) \\
& \frac{d I_{v}(t)}{d t}=\Sigma_{1}^{n} \varphi_{i}(t) S_{v}(t)-\mu_{v}(t) I_{v},
\end{aligned}
$$

where $\beta_{i}(t)=\frac{a_{i}(t) m_{i}(t) I_{v}(t)}{N_{h}(t)}$ and $\varphi_{i}(t)=$ $\frac{a_{i}(t)\left(c_{i}(t) I_{h}(t)+\int_{0}^{1} \tilde{c}_{i}\left(t, \theta^{\prime}\right) R_{h}^{(i)}\left(t, \theta^{\prime}\right) d \theta^{\prime}\right)}{N_{h}(t)}$ with initial condition $\left(P_{1}\right)$ or $\left(P_{2}\right)$.

In the sequel, we will study some mathematical and biological properties of our model (1) simplified as (2) and present the analysis derived.

\section{MAIN RESULTS UNDER INITIAL CONDITION $P_{1}$}

A. Well-posedness of the model (1) with initial condition $P_{1}$ and $g(t, \theta)=(1-k) \gamma(t, \theta)$

Assumption 3.1: Assume that the functions $a, m, \delta, c, k, \gamma(., \theta), \mu_{h}, \tilde{\mu}_{h}, \mu_{v}$ are positive and bounded (as $x, f$ of the case 2 ), for example in $L_{+}^{\infty}(0,+\infty)$. Moreover, $g(t, \theta)=$ $(1-k) \gamma(t, \theta)$.

Assumption 3.2: Assume that the functions $\delta, \mu_{h}, \tilde{\mu}_{h}, \mu_{v}$ are positive, $g=0, e, q=0$ and constant (as $x, f$ of the case 2), and the functions $a, m, \tilde{c}, \gamma, c$ are positive and bounded (as $x, f$ of the case 2), for example in $L_{+}^{\infty}(0,+\infty)$ for $a, m, c$ and $L_{+}^{\infty}(0,+\infty) \times(0,1)$ for $\tilde{c}, \gamma$. Moreover,

$$
\min \left\{x, f, \tilde{\mu}_{h}\right\}-\left[k \gamma+\operatorname{esssup}_{[0,+\infty)}\{\beta\}\right]>0 .
$$

Proposition 3.1: Under Assumptions 3.1. we have $N(t)=S_{h}(t)+I_{h}(t)+\int_{0}^{1} R_{h}(t, \theta) d \theta+$ $S_{v}(t)+I_{v}(t)$ and a suitable differentiation under the integral:

$$
\begin{aligned}
& (e+q)-\left(\max \left\{x, f, \tilde{\mu}_{h}, \sup _{[0,+\infty)}\{\delta\}\right\}\right) N \leq \frac{d N}{d t} \leq \\
& (e+q)-\left(\min \left\{x, f, \mu_{h}\right\}-\left[\begin{array}{c}
k \gamma+\underset{[0,+\infty)}{\operatorname{esssup}}\{\beta\} \\
{[0,}
\end{array}\right) N .\right.
\end{aligned}
$$

Moreover, one could re-write model (1) with initial conditions as the following abstract non autonomous Cauchy problem

$$
\begin{aligned}
u^{\prime}(t)= & A(t, u(t))+V(t) u(t)+H(t, u(t)), t>0, \\
u(0)= & \left(\begin{array}{l}
S_{h}^{0} \\
I_{h}^{0} \\
R_{h}^{0} \\
0 \\
S_{v}^{0} \\
I_{v}^{0}
\end{array}\right) \in X_{0+,}
\end{aligned}
$$

with $[V(t)$.$] bounded and [V(t)]+.H(t,$.$) lo-$ cally Lipschitzian in $t$.

Proof: The proof is obtained by straightforward computations with this strategy. In order to deal with (1)-(initial condition), let us introduce the Banach spaces

$$
X=\mathbb{R} \times \mathbb{R} \times L^{1}(0,1) \times \mathbb{R} \times \mathbb{R} \times \mathbb{R}
$$

and

$$
X_{0}=\mathbb{R} \times \mathbb{R} \times L^{1}(0,1) \times\{0\} \times \mathbb{R} \times \mathbb{R}
$$

endowed with the usual product norm, as well as its positive cone $X_{+}$defined by

$$
\begin{aligned}
X_{+}= & {[0,+\infty) \times[0,+\infty) \times L^{1}(0,1) } \\
& \times[0,+\infty) \times[0,+\infty) \times[0,+\infty)
\end{aligned}
$$

and $X_{0+}=X_{+} \cap X_{0}$. Consider the linear operator $A: D(A) \subset X \rightarrow X$ defined by

$$
D(A)=\mathbb{R}^{2} \times L^{1}(0,1) \times \mathbb{R}^{3}
$$


S Y Tchoumi et al., Mathematical model for acquiring immunity to malaria: a PDE ...

and

$$
A\left(t,\left(\begin{array}{l}
\alpha_{S_{h}} \\
\alpha_{I} \\
z \\
0 \\
\alpha_{S_{v}} \\
\alpha_{I_{v}}
\end{array}\right)\right)=L_{A}(t)
$$

where $R_{h}(t, 1)=0, N_{h}=S_{h}+I_{h}+\int_{0}^{1} R(\theta) d \theta$, $N_{v}=S_{v}+I_{v}$ and:

Case 2: $\left(\Gamma_{h}(t)=e+f(t) N_{h}(t)\right.$ and $\Gamma_{v}(t)=$ $\left.q+x(t) N_{v}(t)\right)$

$$
L_{A}(t)=\left(\begin{array}{l}
-\mu_{h}(t) \alpha_{S_{h}}+\int_{0}^{1} k \gamma(t, y) z(y) d y \\
\quad+f(t) N_{h} \\
-\left(\delta(t)+\tilde{\mu}_{h}(t)\right) \alpha_{I_{h}} \\
-\alpha . \times z^{\prime}-(\gamma(t, .)+\alpha) z \\
-z(0) \\
-\mu_{v}(t) \alpha_{S_{v}}+x(t) N_{v} \\
-\mu_{v}(t) \alpha_{I_{v}} .
\end{array}\right) .
$$

Consider also the non-linear map

$$
F\left(t,\left(\begin{array}{l}
\alpha_{S_{h}} \\
\alpha_{I} \\
z \\
0 \\
\alpha_{S_{v}} \\
\alpha_{I_{v}}
\end{array}\right)\right)=\left(\begin{array}{l}
e-\beta^{*}(t) \alpha_{S_{h}} \\
\beta^{*}(t) \alpha_{S_{h}} \\
\beta^{*}(t)\left[\int_{0}^{\theta} z(y) d y-g . z\right] \\
\delta(t) \alpha_{I_{h}} \\
q+\varphi^{*}(t) \alpha_{S_{v}} \\
\varphi^{*}(t) \alpha_{S_{v}}
\end{array}\right)
$$

with $\beta^{*}(t)=\frac{a(t) m(t) \alpha_{I_{v}}(t)}{\alpha_{S_{h}}(t)+\alpha_{I_{h}}(t)+\int_{0}^{1} z(y) d y}$ and $\varphi^{*}(t)=\frac{a(t)\left(c(t) \alpha_{I_{h}}(t)+\int_{0}^{1} \tilde{c}\left(t, \theta^{\prime}\right) z(y) d y\right)}{\alpha_{S_{h}}(t)+\alpha_{I_{h}}(t)+\int_{0}^{1} z(y) d y}$.

Case 3: $\left(\Gamma_{h}(t)=\Gamma_{h}(:=e)>0\right.$ and $\Gamma_{v}(t)=$ $\Gamma_{v}(:=q)>0$ seen as constants.)

$$
L_{A}(t)=\left(\begin{array}{l}
-\mu_{h}(t) \alpha_{S_{h}}+\int_{0}^{1} k \gamma(t, y) z(y) d y \\
-\left(\delta(t)+\tilde{\mu}_{h}(t)\right) \alpha_{I_{h}} \\
-\alpha . \times z^{\prime}-(\gamma(t, .)+\alpha) z \\
-z(0) \\
-\mu_{v}(t) \alpha_{S_{v}} \\
-\mu_{v}(t) \alpha_{I_{v}}
\end{array}\right)
$$

Consider also the non-linear map

$$
F\left(t,\left(\begin{array}{l}
\alpha_{S_{h}} \\
\alpha_{I} \\
z \\
0 \\
\alpha_{S_{v}} \\
\alpha_{I_{v}}
\end{array}\right)\right)=\left(\begin{array}{l}
\Gamma_{h}-\beta^{*}(t) \alpha_{S_{h}} \\
\beta^{*}(t) \alpha_{S_{h}} \\
\beta^{*}(t)\left[\int_{0}^{\theta} z(y) d y-g . z\right] \\
\delta(t) \alpha_{I_{h}} \\
\Gamma_{v}+\varphi^{*}(t) \alpha_{S_{v}}, \\
\varphi^{*}(t) \alpha_{S_{v}}
\end{array}\right)
$$

with $\beta^{*}(t)=\frac{a(t) m(t) \alpha_{I_{v}}(t)}{\alpha_{S_{h}}(t)+\alpha_{I_{h}}(t)+\int_{0}^{1} z(y) d y}$ and $\varphi^{*}(t)=\frac{a(t)\left(c(t) \alpha_{I_{h}}(t)+\int_{0}^{1} \tilde{c}\left(t, \theta^{\prime}\right) z(y) d y\right)}{\alpha_{S_{h}}(t)+\alpha_{I_{h}}(t)+\int_{0}^{1} z(y) d y}$. In fact, the non linear term can further be broken in two terms: $V(t)$ the linear part describing the initial transmission $\theta=0$ and $H=F-V$ the very non linear part of $F$ corresponding essentially to the horizontal transmission:

$$
V\left(t,\left(\begin{array}{l}
\alpha_{S_{h}} \\
\alpha_{I} \\
z \\
0 \\
\alpha_{S_{v}} \\
\alpha_{I_{v}}
\end{array}\right)\right)=\left(\begin{array}{l}
0 \\
0 \\
0 \\
\delta(t) \alpha_{I_{h}} \\
0 \\
0
\end{array}\right) .
$$

Now identifying $\left(\begin{array}{l}\alpha_{S_{h}} \\ \alpha_{I} \\ z \\ 0 \\ \alpha_{S_{v}} \\ \alpha_{I_{v}}\end{array}\right)$ with $u(t)=\left(\begin{array}{l}\alpha_{S_{h}} \\ \alpha_{I} \\ z \\ \alpha_{S_{v}} \\ \alpha_{I_{v}}\end{array}\right)$, re-writes (1)+initial condition as the following abstract non autonomous Cauchy problem

$$
u^{\prime}(t)=A(t, u(t))+V(t) u(t)+H(t, u(t)), t>0,
$$

$$
u(0)=\left(\begin{array}{l}
S_{h}^{0} \\
I_{h}^{0} \\
R_{h}^{0} \\
0 \\
S_{v}^{0} \\
I_{v}^{0}
\end{array}\right) \in X_{0+}
$$

Remarks 3.1: If we consider $\beta(t)$ with $N_{v}(t)$ at the denominator like Didjou [8] 
instead of $N_{h}$, then Property 3.1 provides the boundedness due to the Gronwall inequality. The choice of the denominator of $\beta(t)$ is an interplay between modeling considerations and the biological explications of the models in literature.

Proposition 3.2: Under Assumptions in Case 2, we have the following:

i) $A$ is an infinitesimal generator of a strongly continuous semigroup $\left(T_{A}(t)\right)_{t>0}$ that is exponentially stable with domain $D(A)$.

ii) $\mathrm{N}$ is bounded.

iii) under more restrictive conditions, one could obtain that $\|V(t)\|<\|\delta\|_{\infty}$ and $(A+V(t))_{t>0}$ generates an evolution family. Moreover the non linear map $F$ is locally Lipschitz-continuous and for all initial condition in $\mathrm{X}$, there exists an interval of time $\left[0, t_{\max }\right)$ in which the problem (1)-(initial condition) has a unique mild solution [26], [20] and is wellposed. Moreover, the mild solution is a global solution on $[0,+\infty)$.

Proof:

1. The existence and uniqueness of the strongly continuous and infinitesimal semigroup of $A$ in the Item i) is proved from theorem A.7 of [17], retrieved by [26] in page 673 .

2. Item ii) and the exponential stability come from $(\mathrm{Pa})$ in Proposition 3.1 and Gromwall inequalities.

3. Item iii) This local solution is bounded on bounded time intervals by the boundedness of the mild solution. Then Theorem A.8 [20], [26] implies that the maximum time interval on which the solution exists is infinite. In other words the mild solution is a global solution on $[0,+\infty)$.

Remarks 3.2: To study the problem (1)(initial condition $P_{2}$ ), one can consider the methods in the work of Chekroun [6].
B. Steady states of model (1) for

$$
g(t, \theta)=(1-k) \gamma(t, \theta) .
$$

Notation 3.1: We set the following:

1- $Y_{0+}=\{X \in C([0,1], \mathbb{R}) \cap C((0,1), \mathbb{R}), X \geq 0\}$;

2- $f=0, x=0, \tilde{c} \equiv c$ is constant, $\tilde{\gamma}=\gamma+\alpha$ and $a, m, \mu_{h}, \mu_{v}, \gamma$ are positive constants;

3 - when it exists, the steady states $X^{*}=\left(S_{h}^{*}, I_{h}^{*}, R_{h}^{*}, S_{v}^{*}, I_{v}^{*}\right)$ is either the disease free equilibrium (DFE) $X^{F}:=$ $\left(S_{h}^{F}, I_{h}^{F}, R_{h}^{F}, S_{v}^{F}, I_{v}^{F}\right)$ or an endemic equilibrium (EE) $X^{E}:=\left(S_{h}^{E}, I_{h}^{E}, R_{h}^{E}, S_{v}^{E}, I_{v}^{E}\right)$;

4- $\tilde{\delta}:=\delta+\tilde{\mu}_{h}, \quad \phi^{*}=\frac{a c\left(I_{h}^{*}+\int_{0}^{1} R_{h}^{*}(\delta) d \theta\right)}{\left.S_{h}^{*}+\int_{0}^{1} R_{h}^{*}(\delta) d \theta\right)+I_{h}}$ and $\beta^{*}=\frac{a m I_{v}^{*}}{\left.S_{h}^{*}+\int_{0}^{1} R_{h}^{*}(\delta) d \theta\right)+I_{h}} ;$

5 - the "next generation operator" $K: Y_{0+} \rightarrow$ $X_{0+}$ such that $\forall R_{h}^{*} \in X_{0+}$ :

$$
\begin{aligned}
& K\left(R_{h}^{*}\right)[\theta]=e^{-\frac{(\alpha+\gamma)}{\alpha} \ln (\theta)} R_{h}^{*}(0) \\
& +V P \int_{0}^{\theta} \frac{\beta^{\star}}{\alpha \theta^{\prime}} e^{-\frac{(\alpha+\gamma)}{\alpha}\left[\ln (\theta)-\ln \left(\theta^{\prime}\right)\right] \int_{0}^{\theta^{\prime}} R_{h}^{*}(\sigma) d \sigma d \theta^{\prime} .}
\end{aligned}
$$

("VP" of $\int_{0}^{\theta} \ldots d \theta^{\prime}$ is the "pricipal value" seen literally as " $\lim _{\epsilon \longrightarrow 0}$ of $\int_{\epsilon}^{\theta} \ldots d \theta^{\prime}$ ".) We could see $K$ as

$$
\begin{aligned}
& K\left(R_{h}^{*}\right)[\theta]=\theta^{-\frac{(\alpha+\gamma)}{\alpha}}\left[R_{h}^{*}(0)+\right. \\
& \left.\frac{\beta^{\star}}{\alpha} \int_{0}^{\theta} \theta^{\prime \frac{\gamma}{\alpha}} \int_{0}^{\theta^{\prime}} R_{h}^{*}(\sigma) d \sigma d \theta^{\prime}\right] .
\end{aligned}
$$

6- Precisely with $\tilde{\beta}=\frac{e+k \gamma \int_{0}^{1} R_{h}^{*}\left(\theta^{\prime}\right) d \theta^{\prime}}{S_{h}^{E}}-\beta^{*}$, $K\left(R_{h}^{*}\right)[\theta]=\theta^{-\frac{(\alpha+\gamma)}{\alpha}}\left[R_{h}^{*}(0)+\right.$ $\left.\frac{1}{\alpha}\left(\frac{e+k \gamma \int_{0}^{1} R_{h}^{*}\left(\theta^{\prime}\right) d \theta^{\prime}}{S_{h}^{E}}-\tilde{\beta}\right) \int_{0}^{\theta} \theta^{\prime} \frac{\gamma}{\alpha} \int_{0}^{\theta^{\prime}} R_{h}^{*}(\sigma) d \sigma d \theta^{\prime}\right]$

7- $r(K)$ is the spectral radius of the next generation operator [10].

8- $\lambda_{1}=I_{h}^{*}+\int_{0}^{1} R_{h}^{*}(\theta) d \theta$ and $\lambda_{2}=I_{v}^{E}$. In the literature, it is common to consider $r(K)$ as an equivalence of the basic reproduction rate. Under the condition on compactness and the supporting properties 
S Y Tchoumi et al., Mathematical model for acquiring immunity to malaria: a PDE ...

of $K$ [10] [Proposition 6.6, page 321], it is possible to prove the existence of the endemic steady states and study the stability of the steady states depending on the sign of $r(K)-1$. .

Some biological remarks on the possible steady states and the basic reproduction threshold is summarized in the following proposition with straightforward computation.

Proposition 3.3: Following the notations above in Notation III-B, we have the following results:

1- Possible steady states seen as $\left\{S_{h}^{*}, I_{h}^{*}, R_{h}^{*}, S_{h}^{*}, I_{v}^{*}\right\}, \quad$ are: $\quad X^{F}=$ $\left\{\frac{e}{\tilde{\beta}}, 0,0, \frac{q}{\tilde{\mu}_{v}}, 0\right\}$ for the disease free equilibrium, and $X^{E}=\left\{\frac{e}{\tilde{\beta}}, I_{h}^{E}, R_{h}^{E}, \frac{q}{\tilde{\mu}_{v}}, I_{v}^{E}\right\}$ for the endemic equilibrium;

2- Endemic steady state $X^{E}$ is such that:

a) $S_{v}^{E}=\frac{q-\mu_{v} I_{v}}{\tilde{\mu}_{v}}$ with $S_{v}^{E}<S_{v}^{F}$;

b) $e+k \gamma \int_{0}^{1} R_{h}^{E}(\delta) d \delta=\mu_{h} S_{h}^{E}+\tilde{\delta} I_{h}^{E}$.

Remarks 3.3: The existence problem of the endemic steady state(s) is a solution to the fixed-point problem $F\left(R_{h}^{E}\right)=R_{h}^{E}$ where $F$ is an operator, in a subspace of $Y_{0+}$.

\section{Numerical Simulations}

In this section, our initial conditions are: $S_{h}(0)=100000$ individuals (humans), $I_{h}(0)=$ 1000 individuals (humans), $R_{h}(0, \theta)=\delta(0) *$ $I_{h}(0)$ individuals (humans), $S_{v}(0)=10000$ mosquitoes, $I_{v}(0)=10000$ mosquitoes. We run the simulation over $T=1500$ days. We set also: $g(t, \theta)=(1-k) \cdot \gamma(t, \theta)$ following (3) and considering individuals living with mosquitoes without clinical malaria by reinforcing premunition, we run the simulations.

A. Simulation for (1)-(initial condition $P_{1}$ ) with $g(t, \theta)=(1-k) \gamma(t, \theta)$ with more infectious humans for a long period of time

In this case, immigration rate in humans is $e=\frac{1000}{59 * 365}$, [5, Table 1 , page 4]. For other parameter values, see Table $\mathrm{I}$ with $\alpha=0.85$ (assumed). After several simulations, all the compartments go to extinction (zero) and there is just nothing we can say further. This type of results is not interesting as seen in the literature ([19] and references therein). The problem (1)-(initial condition $P_{2}$ ) the is more interesting as we see below.

B. Simulations of model (1) with initial condition $P_{2}, g(t, \theta)=(1-0.2466) \gamma(t, \theta)$ and less infectious humans for a long period of time

The immigration rate in humans is given to be $e=\frac{1}{59 * 365}$, [5, Table 1, page 4]. For other values of parameters, see Table I] with $\alpha=$ 0.85 (assumed). Numerical simulations give the following results:

In figures $1 \mathrm{a}, 1 \mathrm{~b}, 1 \mathrm{c}, 1 \mathrm{~d}, \mathrm{Fig} 2$ and other figures not shown here, we observe that the parameters $e$ and $k$ play a great role in the dynamics of the model. We also see that the number of infectious humans could go to zero with a large number of recovered individuals installed for a long time: The disease subsisting in mosquitoes and human and they do not fall sick although they bear the parasite. We see graphically that the proportion $p_{1}$ of recovered individuals with a premunition level $\theta \in\left[\frac{1}{2}, 1\right]$ is greater than the proportion $p_{2}$ of recovered individuals with a premunition level $\theta \in\left[0, \frac{1}{2}\right]$. It suggests that people in areas where malaria is endemic, are more likely to get premunition if they are continuously bitten by mosquitoes.

C. Simulation of model (1) with initial condition $P_{2}$ and $g(t, \theta)=\left(1-\frac{1}{10}\right) \gamma(t, \theta)$

In this case, the immigration rate in humans is $e=\frac{1000}{59 * 365}$ (see Table 1, page 4, [5]). For other parameter values, see Table $[$ with $\alpha=0.85$ (assumed).

In figures $3 \mathrm{a}, 3 \mathrm{~b}, 3 \mathrm{c}, 3 \mathrm{~d}$ and Fig 4 , one could again say that the proportion $p_{1}$ of recovered individuals with a premunition 

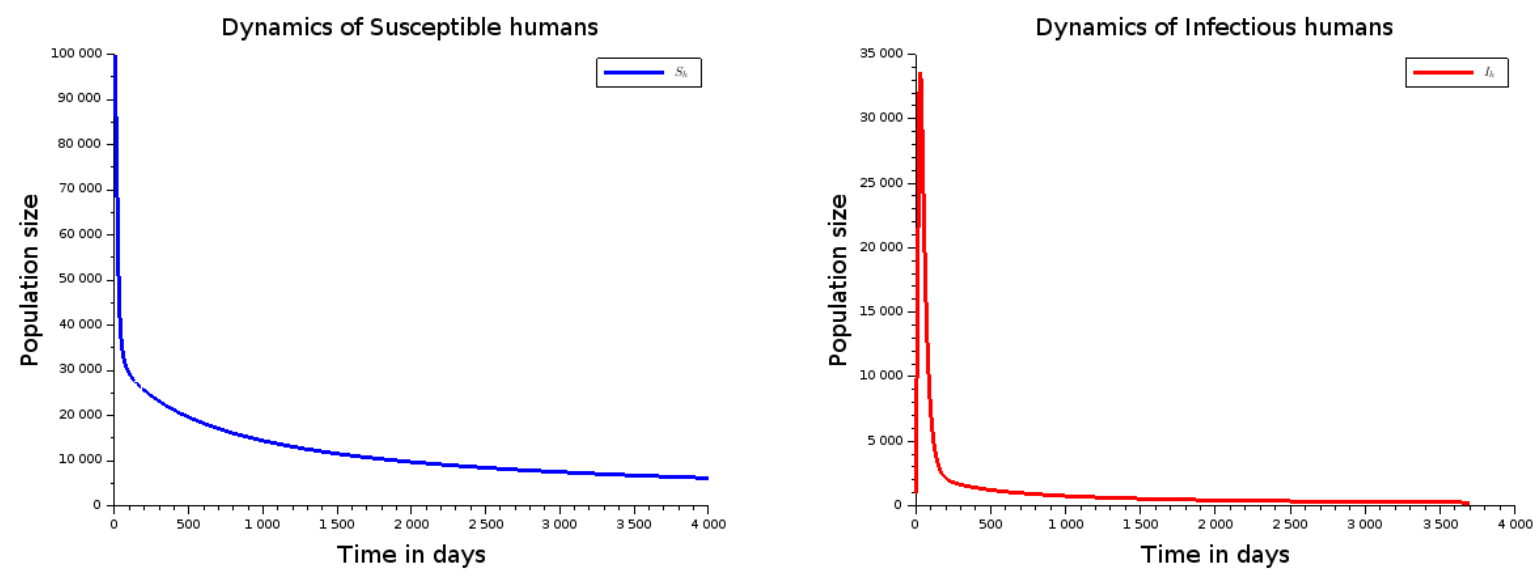

(a) Suceptibles humans,

$\left(e=\frac{1}{59 * 365}, g(t, \theta)=(1-0.2466) \gamma(t, \theta)\right)$

(b) Infectious humans

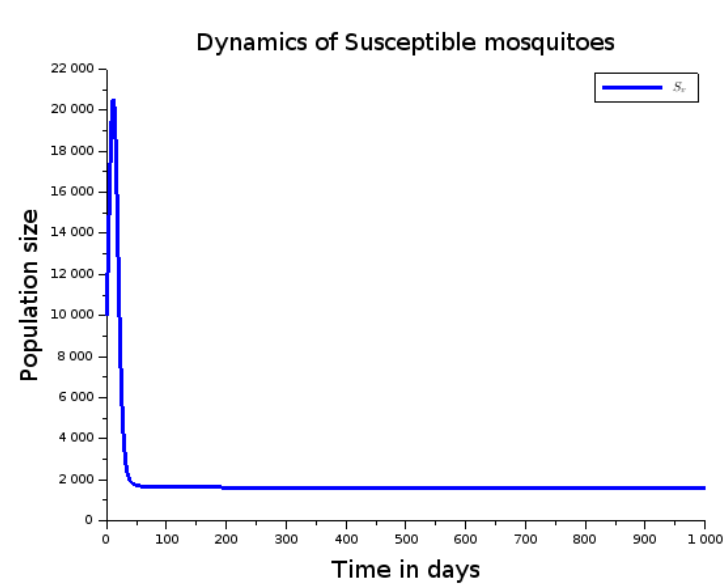

$\left(e=\frac{1}{59 * 365}, g(t, \theta)=(1-0.2466) \gamma(t, \theta)\right)$

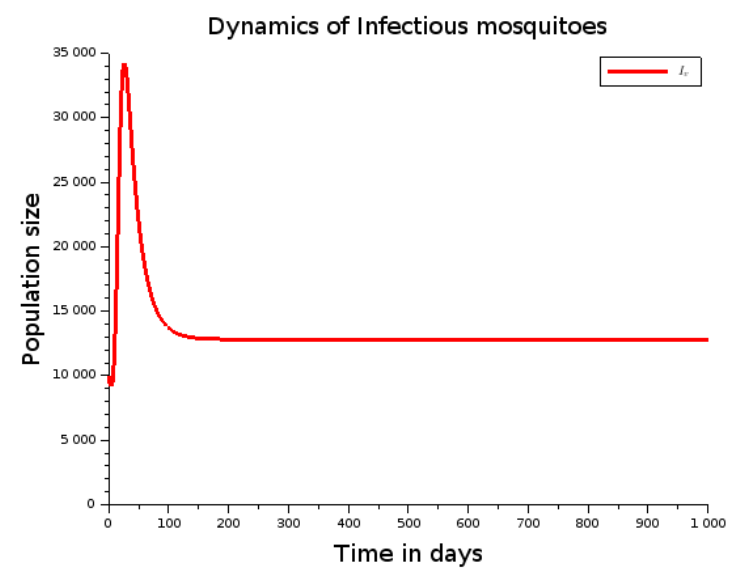

(c) Susceptible mosquitoes

$\left(e=\frac{1}{59 * 365}, g(t, \theta)=(1-0.2466) \gamma(t, \theta)\right)$

(d) Infected mosquitoes

$\left(e=\frac{1}{59 * 365}, g(t, \theta)=(1-0.2466) \gamma(t, \theta)\right)$

Fig. 1: Dynamics of the model (1) using $P_{2}$ with $e=\frac{1}{59 * 365}$, and $g(t, \theta)=(1-0.2466) \gamma(t, \theta)$

level $\theta \in\left[\frac{1}{2} ; 1\right]$ is greater than the proportion $p_{2}$ of recovered individuals with a premunition level $\theta \in\left[0, \frac{1}{2}\right]$, and support the fact that the factor $e$ and $k$ are very important.

\section{Discussion}

a) : A model for naturally acquiring immunity to malaria diseases was studied in the present work. The ability or capacity of a person who acquires natural immunity to live with a relatively low concentration of malaria and resist to falling sick was considered to be the premunition. Susceptibility and death are high during childhood but as children grow, they gradually begin to have intermittent absence of parasitemia, followed by lower density parasitemia, splenomegally and finally premunition. On the other hand, pregnant women especially primigravids (first pregnancy) are highly susceptible to malaria infections and serious diseases since the natural defense 


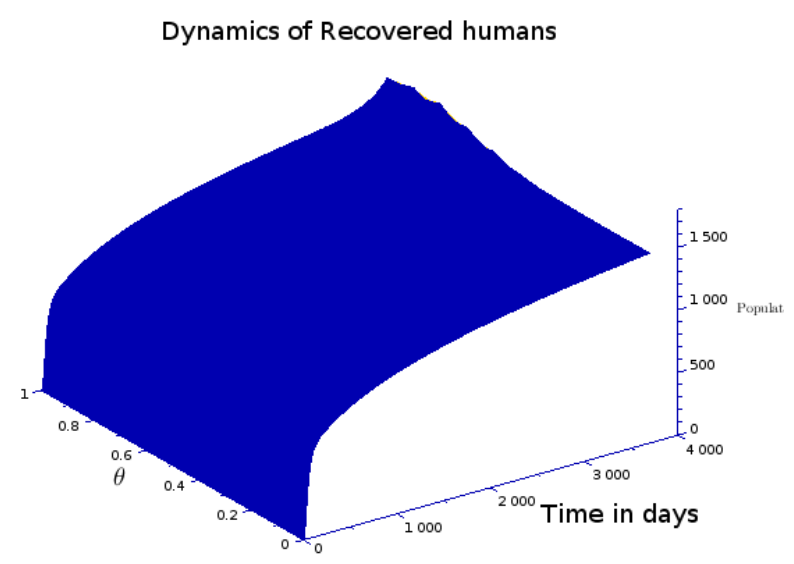

Fig. 2: The number of recovered individuals $\left(e=\frac{1}{59 * 365}, g(t, \theta)=(1-0.2466) \gamma(t, \theta)\right)$

mechanisms are reduced during pregnancy. Adolescents and adults sometimes have parasitemia and occasionally clinical symptoms but their premunition depends on the individual's antibodies. Antibodies as a protective measure can also boost the immunity of an individual to malaria. The pathogen replication cycle assumes a typical viral pathogen that replicates using a machinery of host cells called target cells [16]. The ability of the target cells to fight the disease is what gives premunition to an individual's organism.

b) : In section I we gave a review of previous work concerning the loss of immunity and pointed that this work on acquiring immunity for malaria is a pioneer approach. In section III, we derive an integro-differential equation for acquiring immunity for malaria in humans taking into account all possible strains of the pathogens indexed by $i$. In section III, we obtained three main results from the analysis of the model namely: the wellposedness of the model was established with initial conditions $\mathrm{P} 1$, the generator term $\mathrm{A}$ was bounded and found to be exponentially stable in the domain $D(A)$ defined in Propo- sition 3.1. $N$ was shown to be bounded in Proposition p2. Numerical simulations were done in section IV, with initial conditions $P_{1}$ and $P_{2}$. The results with $\mathrm{P} 1$ was quiet. Simulations with initial conditions P2 gave more interesting results. In subsections IV-B and IV-C we suggested that the premunition is a continuous process because the relative resistance to a low level of parasite seems to increase with time in an endemic situation. It is clear that the level of premunition is somehow a probability with a possibility to suffer from the severe malaria disease if other negative factors are dramatically considered (physiological lacks, physical or psychological injuries ...) even if the level of premunition is high.

c) : The use of bed nets(represented by $k)$ is recommended without interruption to avoid a severe disease due to a possible loss of immunity. At least, a partial immunity could be acquired if the bed net is not properly used and rigorous. We observed from subsections IV-B and IV-C, that more recovered people in endemic areas seem to have a high degree of immune responsiveness after being continuously exposed to mosquitoes bites. This is why Obi et al. [19] said that "humans repeated infection by Plasmodium falciparum induce a progressive modulation of the immune response, eventually leading to an anti-parasite immunity characteristic of premunition". Beside that, we observed in figures 2 and 4 a relatively increase in the number of recovered individuals with temporary immunity, for a long period of time (about 1000 units).

\section{CONCLUSION}

a) : In this paper, we have analyzed the consideration of continuous acquisition of immunity (from level 0 to level 1). Our paper confirms that living in malaria endemic areas implies a premunition in the body of people exposed to infected mosquitoes bites. If mosquitoes bites stops for a long time, 


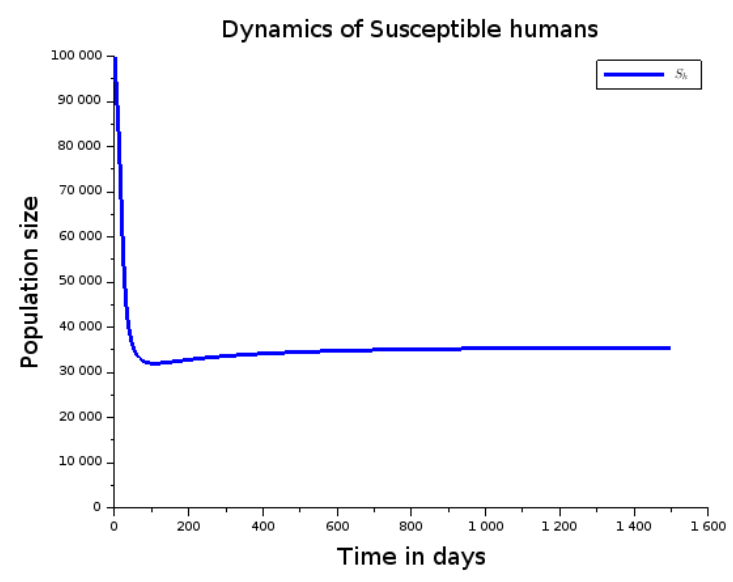

(a) Suceptibles humans,

$\left(e=\frac{1000}{59 * 365}, g(t, \theta)=\left(1-\frac{1}{10}\right) \gamma(t, \theta)\right)$

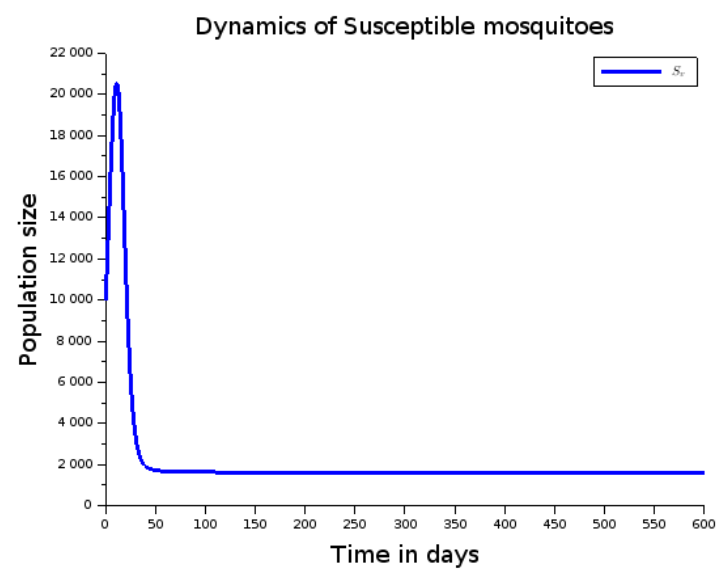

(c) Susceptible mosquitoes

$\left(e=\frac{1000}{59 * 365}, g(t, \theta)=\left(1-\frac{1}{10}\right) \gamma(t, \theta)\right)$

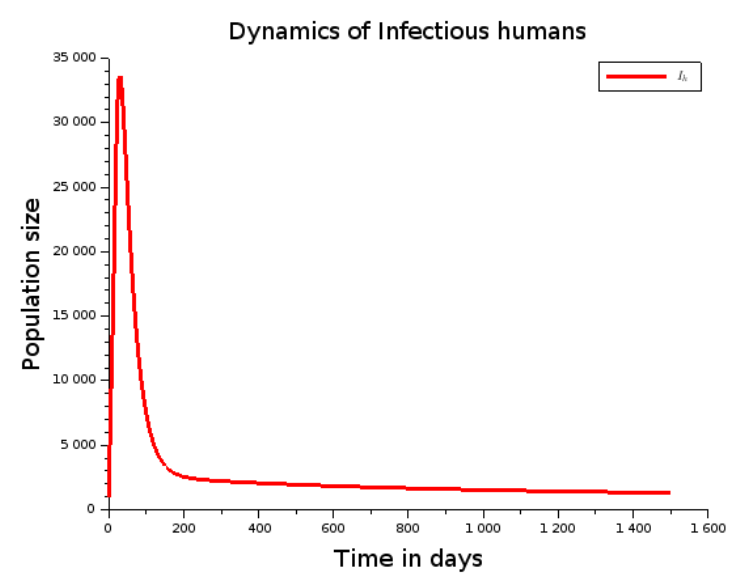

(b) Infectious humans

$\left(e=\frac{1000}{59 * 365}, g(t, \theta)=\left(1-\frac{1}{10}\right) \gamma(t, \theta)\right)$

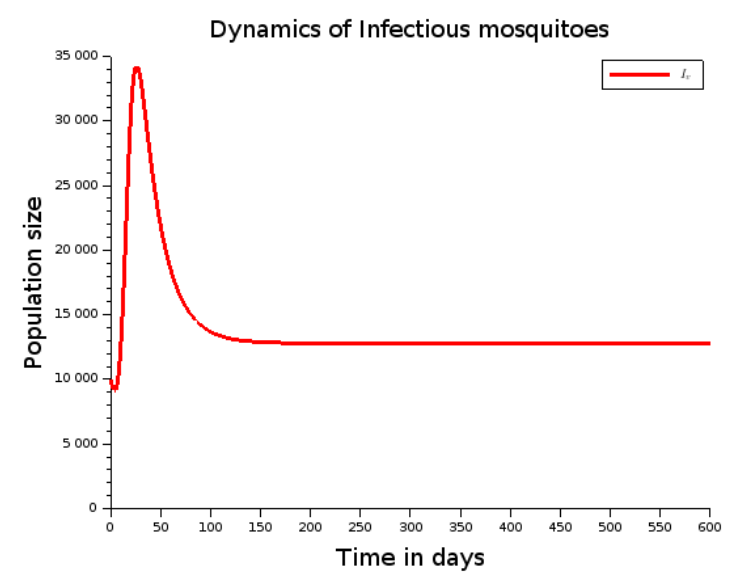

(d) Infected mosquitoes

$\left(e=\frac{1000}{59 * 365}, g(t, \theta)=\left(1-\frac{1}{10}\right) \gamma(t, \theta)\right)$

Fig. 3: Dynamics of the model (1)- $P_{2}$ with $\left(e=\frac{1000}{59 * 365}, g(t, \theta)=\left(1-\frac{1}{10}\right) \gamma(t, \theta)\right)$

susceptible can loose their relative immunity to malaria disease. People should be conscious and rigorous in the using bed nets especially in malaria endemic areas to avoid super infection which and failure of premunition to malaria disease. A perspective of this work could be to include a space variable in the model to target places with high risk of infection (e.g water pools, ...). In fact, the choice of $g$ depends on the context one has. To find the right function $g$, one needs to solve a kind of inverse problem from the data. That is a huge perspective where the least square method could be used. Finally, it's common to see in malaria endemic areas that the population (in adulthood likely more than five years old) could live mainly as "recovered" even within the presence of heavy parasitemia and this is the reason why public health strategies in 


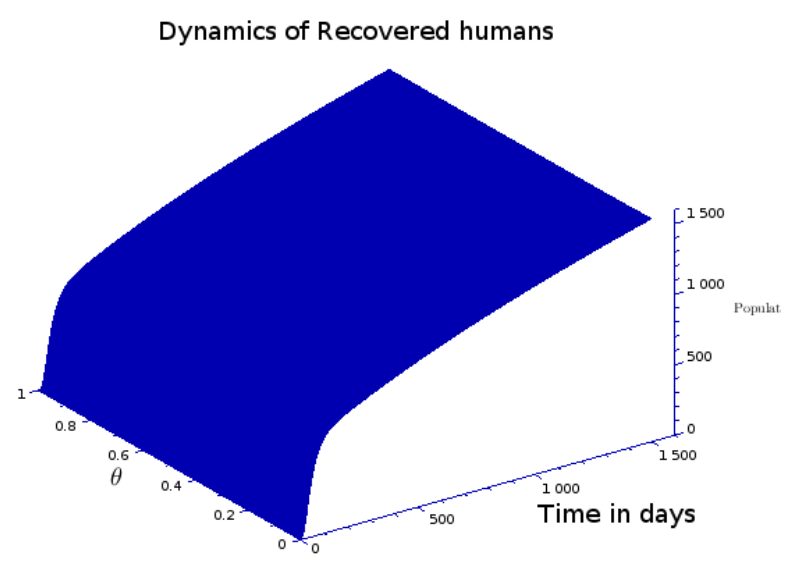

Fig. 4: The number of recovered individuals $\left(e=\frac{1000}{59 * 365}, g(t, \theta)=\left(1-\frac{1}{10}\right) \gamma(t, \theta)\right)$

high endemicity region of malaria, concerns children from 0 to 5 years old and pregnant women: a new model with age of infection is then more relevant.

\section{REFERENCES}

[1] R. Anguelov, Y. Dumont, J. Lubuma and E. Mureithi. Stability Analysis and Dynamics Preserving Nonstandard Finite Difference Schemes for a Malaria Model, Mathematical Population Studies: An International Journal of Mathematical Demography, 20:2, (2013), 101-122.

[2] C. Antonio Nkondjio., C. Ndo, F. Njiokou, J. D. Bigoga, P. Awono-Ambene, J. Etang, A. S. Ekobo 4 and C. S. Wondji. Review of malaria situation in Cameroon: technical viewpoint on challenges and prospects for disease elimination, Parasites Vectors, https://doi.org/10.1186/s13071-019-3753-8 , 12:501, (2019).

[3] J.K. Aron. Mathematical modeling of immunity to malaria. Math. Biosci., 90, 385-396, (1988).

[4] O.J.T Briet. A simple method for calculating mosquito mortality rates, correcting for seasonal variations in recruitment. Med. Vet. Entomol. 16, (2002), pp22-27.

[5] B. Buonomo. Analysis of a malaria model with mosquito host choice and bed-net control. International Journal of Biomathematics (2013).

[6] A. Chekroun and T. Kuniya, An infection agespace structured SIR epidemic model with Neumann boundary condition, Appl. Anal. (2018), https://doi $\quad . o r g \quad / 10 \quad .1080 \quad / 00036811 \quad$ (2018), 1551997.

[7] N. Chitnis, J. M. Hyman and J. M. Cushing. Determining Important Parameters in the Spread of Malaria Through the Sensitivity Analysis of a Mathematical Model, Bulletin of Mathematical Biology (2008) 70: 1272-1296, DOI 10.1007/s11538-008-9299-0.

[8] R. Djidjou-Demasse, G. J. Abiodun, A.M. Adeola, J. O. Botai. A malaria transmission model with seasonal mosquito life-history traits, BioRxiv, doi: https://doi.org/10.1101/377184, 2018.

[9] D. L. Doolan, C. Dobano and J. K. Baird. Acquired Immunity to Malaria. Clinical Microbiology Reviews, 22(1), (Jan 2009), pp13-36; Full text at http://cmr.asm.org/cgi/reprint/22/1/13

[10] H. Inaba, Chapter 6 (Age-structured SIR Model). Age-structured population dynamics in demography and epidemiology, (2017), pp 287-331, Springer.

[11] J. Langhorne, F. M. Ndungu, A.-M. Sponaas, K. Marsh. Immunity to malaria: more questions than answers, Nature Immunology volume 9, (2008) pp725-732.

[12] S. Mandal, R.R. Sarkar, S. Sinha, Mathematical models of Malaria, a review. Malaria Journal, (2011), 10:202.

[13] Immunity (malaria), https://www.malariasite.com/immunity/ (Site Last Updated on March 16, 2019).

[14] Malaria Foundation International (MFI). (assessed on 5/5/2020: empty, but cited by J. M. Tchuenche et al. in doi:10.1093/imammb/dqq017 , 2010) Available at http://www.malaria.org.

[15] K. Marsh. Malaria disaster in Africa. The Lancet, 352, 924, 1998.

[16] M. Martcheva, N. Tuncer, C. Mary. Coupling within-host and between-host infectious diseases models. Biomath. (2015), https://doi .org /10 .11145 /jbiomath.2015.

[17] M. Matsui, and F. Takeo, Chaotic semigroups generated by certain differential operators of order 1. SUT Journal of Mathematics 37, 1, (2001), pp 51-67.

[18] G. A. Ngwa and W. S. Shu, A mathematical model for endemic malaria with variable human and mosquito populations. Mathematical and Computer Modelling, 32(7-8), (2000), pp747-763.

[19] R. K. Obi, C. C. Okangba, F. C. Nwanebu, U. U. Ndubuisi and N. M. Orji, Premunition in Plasmodium falciparum malaria, African Journal of Biotechnology Vol. 9(10), pp. 1397-1401, 8 March, 2010

[20] A. Pazy. Semigroups of Linear Operators and Applications to Partial Differential Equations, Applied Mathematical Sciences vol. 44, SpringerVerlag, New York, 1983. 
SY Tchoumi et al., Mathematical model for acquiring immunity to malaria: a PDE ...

[21] M. Pinkevych, J. Petravic, K. Chelimo, J. W. Kazura, A. M. Moormann and M. P. Davenport. The Dynamics of Naturally Acquired Immunity to Plasmodium falciparum Infection, PLoS Comput Biolv.8 (10); (2012).

[22] D. Raoult, Epidémies: vrais dangers et fausses alertes, Éditions Michel Lafon, 2020.

[23] J. M. Tchuenche, D. Chan. A. Matthews and G. Mayer. A mathematical model for antimalarial drug resistance, Mathematical Medicine and Biology, (2010), doi:10.1093/imammb/dqq017.

[24] A. Perasso, Dynamique des populations - Étude d'une propagation d'épidémie, Sujet de projet -
MAP431, Ecole Polytechnique, 2009.

[25] A.-M. Pulkki-Brännström, C. Wolff, N. Brännström and J. Skordis-Worrall. Cost and cost effectiveness of long-lasting insecticidetreated bed nets - a model-based analysis, Cost Effectiveness and Resource Allocation, (2012), 10:5.

[26] N. Ziyadi, S. Boulite and M. Lhassan Hbid. Mathematical of a PDE epidemiological model applied to Scrapie transmission, Comm. on Pure and Appl. An., Volume 7, Number 3, pp. 659-675, May 2008. 